\title{
Roberto Bolaño y sus comienzos literarios: El infrarrealismo entre realidad y ficción
}

\author{
Roberto Bolaño and his literary beginnings: Infrarrealism, \\ between reality and fiction
}

\author{
Chiara Bolognese \\ Universidad Autónoma de Barcelona. Barcelona, España. \\ chiara.bolognese@uab.cat
}

工 vida y la obra del autor chileno Roberto Bolaño evidenciaron constanintentará mostrar que su escritura fue innovadora ya desde sus comienzos como escritor en México, cuando literatura y existencia se fundieron sin ninguna hesitación. Ello permitirá destacar también el papel determinante que tuvo el país centroamericano en su formación cultural y en su producción posterior.

Bolaño protagonizó la bohemia mexicana de los años setenta poco después de llegar al D.F. con su familia, en 1968. Allí, muy pronto, se sintió atraído por el anticonvencionalismo, y por todo lo que se mostraba en contra del sistema de valores dominantes. Éste es un interés que el escritor primero transformó en acción literaria, dando lugar a un proyecto que fue de vida al tiempo que estético, y que, posteriormente, trasladó a la ficción, siendo el mundo de la rebelión y de la contracultura uno de los temas que vertebran sus textos. Fue entonces cuando fundó en 1976 con Mario Santiago el movimiento infrarrealista, posicionándose en el ámbito de la poesía alternativa en la época en la que dominaba el magisterio de Octavio Paz. Y es precisamente para oponerse a Paz por lo que los creadores pretendían producir una literatura nueva y totalmente distinta.

El movimiento consistía en un grupo posvanguardista que celebraba el profundo amor por la poesía, fusionado con una actitud contestataria frente a todo lo socialmente establecido y cimentado sobre una estrecha amistad entre sus dos fundadores y los demás miembros.

La necesidad de la creación de un nuevo grupo artístico nació en relación con el hecho de que en la época los talleres literarios iban adquiriendo un papel 
fundamental dentro del mundo académico y cultural. Muchos de los jóvenes que luego pasaron a formar parte del infrarrealismo iban al Taller de Poesía de la Universidad Autónoma de México impartido por Juan Bañuelos. Los estudiantes no apreciaban la manera de acercarse a la poesía propuesta por el director, la cual, por otra parte, no era sino el reflejo de cierta cerrazón de la cultura oficial del momento. A raíz de eso, llegaron a proponer y obtener el cese de la actividad, abriendo así el paso al nacimiento del infrarrealismo.

Bolaño no iba al taller porque nunca se matriculó en ninguna carrera, ni circuló por los ambientes de la academia. El autor conoció a Mario Santiago en una tertulia en el café La Habana, un lugar que más tarde aparece en Los detectives salvajes como el café Quito, sede de las muchas reuniones de los jóvenes protagonistas. En este sentido, son interesantes las declaraciones que hace el mismo Bolaño durante una entrevista con la poeta y novelista mexicana Carmen Boullosa:

El infrarrealismo fue una especie de Dadá a la mexicana. En algún momento hubo mucha gente, no sólo poetas, sino pintores y sobre todo vagos y ociosos, que se consideraron a sí mismos como infrarrealistas, pero en realidad el grupo sólo lo integrábamos dos personas, Mario Santiago y yo. Ambos nos vinimos a Europa en 1977. Después de algunas aventuras desastrosas, una noche en la estación de trenes de Port Vendres, en el Rosellón, muy cerca [...] de la estación de trenes de Perpignan, decidimos que el grupo como tal se había acabado (Bolaño, en Manzoni, 2002: 112)ํ.

Los infrarrealistas fundaron primero la revista Correspondencia Infra, revista menstrual del movimiento infrarrealista, que sacó muy pocos ejemplares, y sin embargo es sumamente relevante porque en sus páginas se publicó el Primer manifiesto infrarrealista, redactado por el mismo Bolaño en la casa de otro joven poeta chileno, Bruno Montané. El texto evidencia que las ideas que darían forma al proyecto literario del autor ya estaban delineadas desde sus primeros versos y planteamientos teóricos. Éstas luego se desarrollaron, profundizaron

${ }^{1}$ En Los detectives salvajes, justamente en Port Vendres Arturo se despide de Ulises con las siguientes palabras: "se me hace que ésta es la última vez que nos vemos" (1988: 269). Sin embargo, en dicha novela el principio del agotamiento del real visceralismo se da cuando, en 1977, Müller y Belano, ya en Europa, mandan una carta de renuncia a sus compañeros (cfr. pp. 243-244). Acerca del parecido entre el infrarrealismo y el dadaísmo, confróntense también las siguientes palabras de Maples Arce sobre la relación entre su movimiento y el mencionado dadaísmo: "Con este vocablo dorado: estridentismo, hago una transcripción de los rótulos dadá, que están hechos de nada, para combatir la 'nada oficial de libros, exposiciones y teatro'. En síntesis una fuerza radical opuesta contra el conservatismo solidario de una colectividad anquilosada" (Maples Arce, en Schwartz, 1991: 167). 
y enriquecieron en sus obras sucesivas. El manifiesto muestra que el hilo que siempre está presente marcando toda la producción bolañana está representado por el tema del fracaso de las utopías, como exponen las siguientes palabras: "soñábamos con la utopía y nos despertamos gritando"2.

El veinteañero Bolaño se sentía fascinado por la realidad marginal, por la alcantarilla de la realidad -la infrarrealidad, justamente- de quienes lo han arriesgado todo, y tal vez lo han perdido todo: los desesperados de y por la literatura, como también lo serán la mayoría de los personajes de sus obras posteriores. Se trata de individuos que encarnan la fusión de arte y vida, enfrentándose con la existencia con realismo pero desde dentro, visceralmente: "Vamos a meternos de cabeza en todas las trabas humanas, de modo tal que las cosas empiecen a moverse dentro de uno mismo, una visión alucinante del hombre". De allí, posiblemente, el nombre de real visceralismo que elige Bolaño para el movimiento que constituye el eje de su novela Los detectives salvajes.

La función principal del infrarrealismo era la de derrocar la cultura oficial a la que retrataban de la siguiente manera:

cortinas de agua, cemento o lata, separan una maquinaria cultural, a la que lo mismo le da servir de conciencia o culo de la clase dominante, de un acontecer cultural vivo, fregado, en constante muerte o nacimiento, ignorante de gran parte de la historia y las bellas artes [...] cuerpo que por lo pronto experimenta en sí mismo sensaciones nuevas, producto de una época en que nos acercamos a $200 \mathrm{kph}$ al cagadero o a la revolución (Bolaño, 1977).

$\mathrm{Y}$ es en esta actitud que los infrarrealistas recogen el legado del estridentismo de Manuel Maples Arce, de cuyo manifiesto reproducen el tono. Estos jóvenes poetas compartían la visión del arte a la que invitaban los estridentistas, quienes rechazaban con ahínco todo lo que proponían los intelectuales reunidos alrededor de la revista Contemporáneos, como muestran las primeras líneas de su segundo manifiesto:

Irreverentes, afirmales, convencidos, excitamos a la juventud intelectual del estado de Puebla, a los no contaminados de reaccionarismo letárgico, a los no identificados con el sentir medio colectivo del público [...] para que vengan a engrosar las filas triunfales del estridentismo (Maples Arce, 1991: 170)33.

2 El Manifiesto infrarrealista está disponible (en línea) en www.infrarrealismo.com. Consulta: 21/09/ 2009.

${ }^{3}$ El manifiesto sigue con la invitación a alejarse de la "ranciolatría ideológica" para alcanzar un arte auténtico que consienta "que la poesía sea poesía de verdad". 
Los estridentistas fueron muy radicales en su postura, tal y como queda plasmado en la conclusión del manifiesto que dice: "ser estridentista es ser hombre" (Maples Arce, 1991: 171), con clara referencia a quienes, según ellos, no son hombres sino títeres sometidos al establishment.

$\mathrm{Y}$, de forma parecida, los infrarrealistas fueron jóvenes contestatarios que veían en la poesía "las herramientas para la subversión cotidiana" (Bolaño, 1977). Carmen Boullosa, en la ya citada entrevista, los describe con claridad, proporcionando, además, la visión de quien vivía la situación desde el otro lado de la barricada:

Eran el terror del mundo literario. Yo entonces formaba parte de los solemnes [...] me gustaban los formularios de lecturas de poesía, cócteles, esas cosas absurdas llenas de códigos que de alguna manera me sujetaban, y ustedes [le está escribiendo a Bolaño] eran los terroristas también de estos formularios. Antes de comenzar mi primera lectura de poemas [...] me encomendé a Dios -en quien no creía [...]- para que por favor no fueran a aparecer los infras [...] los infras podían aparecer, irrumpir a media sesión y llamarme tonta (Boullosa, en Manzini, 2002: 112).

Bolaño, Santiago y los demás infrarrealistas se convirtieron, como reconoce Boullosa, en unos revolucionarios temidos por todos, sus figuras empezaron a teñirse de leyenda peligrosa, tal vez pasando a encarnar a los soles negros del cuento que reproduce Bolaño al principio de su manifiesto.

Cabe decir que este texto evoca también a otros "revolucionarios" del mundo cultural de la época. Ya desde su título, "Déjenlo todo, nuevamente", delata una clara filiación surrealista, teniendo en cuenta que André Breton escribió un ensayo titulado "Abandonadlo todo", recogido en Los pasos perdidos, al cual hace implícita referencia. Asimismo, las ideas revolucionarias, de subversión de la realidad que se proponen con las palabras "nuestra ética es la Revolución, nuestra estética la Vida: una-sola-cosa” (Bolaño, 1977), traen a la memoria la revista que el maestro francés fundó en 1930, cuyo título era "Le Surréalisme au service de la Révolution”“" La cercanía fue fortalecida también porque Breton mismo vivió en México una temporada y allí conoció a exponentes importantes del mundo de la cultura, como Trotsky ${ }^{5}$ y Diego Rivera.

Parece bastante claro que Bolaño se identificó, en sus veinte años, con las

\footnotetext{
${ }^{4}$ Una voluntad análoga es la que expresaban los estridentistas que trataron de "aliar la creación estética a la revolución" (Schwartz, 1991: 159).

${ }^{5}$ No puede ser una casualidad que mucho después, en Los detectives salvajes, los real visceralistas encuentran a la sobrina de éste, Verónica Volkow.
} 
ideas del surrealista francés, y, en efecto, escribe en un texto recogido en Entre Paréntesis: "Breton habló de la necesidad de que el surrealismo pasara a la clandestinidad, se sumergiera en las cloacas de las ciudades y de las bibliotecas [...] LA HORA DE SENTAR CABEZA NO LLEGARÁ JAMÁS” (Bolaño, 2004: 93). Estas palabras son un claro llamado a sumergirse en la infrarrealidad de la cual el autor iba hablando en la época de su juventud mexicana. Se trata de unas convicciones que los llevaron a una poesía de rebeldía, cuyos cuestionamientos fundamentales están esbozados en las preguntas del manifiesto cuando se interrogan acerca de quienes los rodean: "¿Y la buena cultura burguesa? ¿Y la academia y los incendiarios? ¿Y las vanguardias y sus retaguardias?” (Bolaño, 1977).

En este sentido, los infrarrealistas declaran su deseo de cercanía con los nuevos grupos posvanguardistas y, en particular, su deuda con los peruanos de Hora Zero: "Nos anteceden las MIL VANGUARDIAS DESCUARTIZADAS EN LOS SESENTAS [...] Nos antecede Hora Zero" (Bolaño, 1977). Estos escritores abogaban por una poesía nueva, liberada del conformismo y de las coerciones políticas, y sostenían que, después de César Vallejo, la poesía peruana había dejado de producir obras literariamente importantes. La vinculación entre los mexicanos y los peruanos es evidente, tanto que en la página web oficial del movimiento infrarrealista se incluye el manifiesto de los Horazerianos, firmado por Juan Ramírez Ruiz y Jorge Pimentel, en el que se dice que "a nosotros se nos ha entregado una catástrofe para poetizarla"6, y nada resulta más adecuado que estas palabras para describir la literatura de Bolaño, que quiere poetizar la realidad de los márgenes y de los marginados.

La elección de actuar en grupos y de hermanarse entre ellos es, además, una actitud típica de los vanguardistas y posvanguardistas que ven así reforzado su empeño. $Y$ de la siguiente manera explican los infrarrealistas la razón por reproducir dicho manifiesto: "pese a diferencias históricas, geográficas, literarias, etcétera, es parte de la poesía rebelde de América del Siglo XX, movimiento cuyas expresiones más evidentes son los beat estadounidenses, los horazerianos de Perú y los infrarrealistas mexicanos" (Bolaño, 1977). Y, en efecto, en el prólogo de Muchachos desnudos bajo el arcoiris de fuego, antología que recoge algunos poemas de Bolaño, Miguel Donoso Pareja dice que ellos, junto a los horazerianos y a otros grupos vanguardistas, abogaban por la "libertad de expresión, [el] rechazo de toda la retórica, [la] destrucción de lo viejo, [la] construcción de lo nuevo" (Donoso, 1979: 26).

Por otra parte, las revolucionarias ideas del infrarrealismo fueron propicia-

\footnotetext{
${ }^{6}$ Página web del movimiento: www.infrarrealismo.com. Consulta: 21/04/2005.
} 
das por la angustia, profunda y justificada, debida a la situación histórica compleja y en fase de cambio que caracterizaba a la sociedad mexicana de los años setenta; una preocupación que tal vez se agudizó porque, poco después de llegar Bolaño al DF, se produjo la matanza de Tlatelolco, acontecimiento al que se hace alusión de paso en los versos del Manifiesto:

Las matanzas, los nuevos campos de concentración [...] Son tiempos duros para la poesía, dicen algunos, tomando té, escuchando música [...] Son tiempos duros para el hombre, decimos nosotros, volviendo a las barricadas después de una jornada llena de mierda y gases lacrimógenos (Bolaño, 1977).

Estas palabras muestran de nuevo una cercanía entre la actitud de los infrarrealistas y la de los estridentistas, quienes propugnaban en particular la idea de una poesía que no fuera sólo escritura, sino más bien una actitud frente a la vida $^{7}$, y en esta óptica define el autor las obras Andamios interiores y Poemas interdictos de Maples Arce como "libros que son útiles [...] para comenzar a ver de una manera diferente la tradición de la poesía mexicana” (Bolaño, 1976a: 49).

Sin embargo, es justo en 1976 cuando Bolaño acusa a los miembros de este movimiento de abandonar la batalla: "En México los estridentistas se van, los "contemporáneos" se quedan, la paz vuelve a casa" (1976a: 49). En ello se puede notar algo de ironía hacia el grupo de poetas más conformistas, los "poetas funcionarios", reunidos bajo el poder de Octavio Paz, quien, junto con Efraín Huerta, fue responsable de la revista Taller (1938-41), continuadora de las tendencias de otra revista, Contemporáneos (1928-31), que promovía una fuerte idea de cosmopolitismo, así como de un cierto intelectualismo (Fernández, 1987: 71), que debía de desagradar a Bolaño. A Taller le siguió Plural, que se convirtió en un punto de referencia obligado para la cultura del país y, más en general, para la cultura en lengua española. Allí Bolaño mismo publicó algunos artículos que en estas páginas se citan.

A pesar de la crítica a los estridentistas arriba expuesta, Bolaño les reconoce el legado sustancial de ruptura y de compromiso que éstos dejaron para las generaciones siguientes: "Los estridentistas no pudieron sostener esas barricadas ácidas de la nueva poesía, pero nos enseñaron más de una cosa sobre los adoquines" (1976a: 49), se lee pocas líneas después en el mismo texto. Y es que el infrarrealismo es la encarnación de lo que tenían que decir las vanguardias

${ }^{7}$ Maples Arce y sus seguidores llevaron un aire de cambio a la cultura de su país, que se concretó más en la disposición frente al mundo que en la efectiva producción artística. Cabe recordar que el estridentismo celebraba el trabajo en las fábricas y exaltaba a las masas obreras, dos temas que cobraron más relevancia en México después de la revolución (1910-1917). 
nuevas después del fracaso de todos los sueños.

Otro aspecto reseñable del Manifiesto infrarrealista es el hecho de que presenta al poeta como héroe:

Nuestros parientes más cercanos: los francotiradores, los llaneros solitarios que asolan los cafés de chinos de latinoamérica [sic.], los destazados en supermarkets, en sus tremendas disyuntivas individuo-colectividad; la impotencia de la acción y la búsqueda (a niveles individuales o bien enfangados en contradicciones estéticas) de la acción poética (Bolaño: 1977).

Se trata más bien de un héroe fracasado, ya que no tiene ni mitos ni metas, y que, sin embargo, invita a lanzarse a la aventura: "el poeta como un héroe develador de héroes [...] si el poeta está inmiscuido, el lector tendrá que inmiscuirse" (Bolaño: 1977). Se aprecia, por lo tanto, la coherencia de Bolaño, quien, ya desde el principio de su actividad literaria, proponía la figura de un artista buscando la intemperie, que quiere vivir en ella, tal y como lo hizo Arthur Rimbaud, su mentor más importante, cuya presencia desdibujada vertebra la literatura del autor. Se crea la figura de un poeta marginado, que deambula por esta infrarrealidad que nace, en palabras del propio Bolaño, del choque entre "formas de vida y formas de muerte [que] se pasean cotidianamente por la retina" (Bolaño: 1977).

Es justamente el valor de la tríada constituida por la literatura, la marginación y la muerte el que salva a los derrotados de Bolaño, que nunca se retractan y siempre se mantienen firmes en su compromiso con la vida y con la escritura. El resultado fue un tipo de poesía (y de narrativa, más tarde) que se dirigía inevitablemente hacia el abismo, haciendo incursión en lo porno, la locura, la marginación, la homosexualidad y la violencia: la mayoría de los textos del chileno están protagonizados por los habitantes del caos del infierno, y muchas veces se trata, en particular, del infierno mexicano de la época que abarca desde los años setenta hasta la actualidad.

Es una literatura de derrotados que se enmarca en la poética del fracaso y de la supervivencia; son palabras escritas por quienes intentaron hacer la revolución y no lo lograron, y que bien quedan representados en estos nombres que menciona el personaje Arturo Belano -alter ego del autor- en el cuento "Carnet de baile":

Los hijos de Walt Whitman, de José Martí, de Violeta Parra; desollados, olvidados, en fosas comunes, en el fondo del mar, sus huesos mezclados en un destino troyano que espanta a los supervivientes [...] Pienso en Beltrán Mo- 
rales, pienso en Rodrigo Lira, pienso en Mario Santiago, pienso en Reinaldo Arenas. Pienso en los poetas muertos en el potro de tortura, en los muertos de sida, de sobredosis (Bolaño, 2001: 215).

Como ya se ha sugerido al principio, Bolaño utilizó muchos episodios de su primera "época rebelde" para armar su ficción. En particular esto se nota en las aventuras de los poetas realvisceralistas mexicanos, protagonistas de Los detectives salvajes, quienes están muy orgullosos de pertenecer a la otra cara del universo cultural y se dedican a la vida bohemia típica de cierta clase de artista y de intelectual, suscitando el desprecio de "los integrados". En este sentido puede ser ejemplificativa la definición que Carlos Monsiváis -ficcionalizado en el texto- da de Lima y Belano, poco después de conocerlos:

dos jóvenes [...] obstinados en no reconocerle a Paz ningún mérito, con una terquedad infantil, no me gusta porque no me gusta, capaces de negar lo evidente $[\ldots]$ sin $[\ldots]$ talento [...] ni argumentos de peso, ni originalidad en sus planteamientos. Dos perdidos, dos extraviados (Bolaño, 1998: 160) ${ }^{8}$.

En efecto, nadie parece considerar los proyectos literarios de los protagonistas. El único que de verdad está interesado en conocer en detalle los planteamientos de los dos fundadores del realvisceralismo, Ulises Lima y Arturo Belano, es el joven estudiante Juan García Madero, al cual, ya desde el principio, se le hace patente el hecho de que sus compañeros realvisceralistas viven la existencia en su totalidad. Ellos disfrutaban de los placeres del sexo, del alcohol y de lo prohibido, tal y como lo hacían los infrarrealistas, cuyo manifiesto invocaba "la experiencia disparada, estructuras que se van devorando a sí mismas, contradicciones locas" (Bolaño: 1977). En la misma línea, los jefes del movimiento mantienen una actitud peculiar frente a la realidad y a la creación literaria: "una de las premisas para escribir poesía preconizadas por el realismo visceral [...] era la desconexión transitoria de un cierto tipo de realidad" (Bolaño, 1998: 19), declara García Madero. Por lo tanto, el primer paso para ser realvisceralista -es decir, poeta auténtico- consiste en alejarse o, mejor, transgredir la realidad del conformismo cultural, como profesaron e hicieron los infrarrealistas, fundiendo su búsqueda literaria y vital.

\footnotetext{
${ }^{8}$ Tal vez se pueda leer en estas palabras de Monsiváis una revancha (ficcional) sobre los ataques y las críticas que los poetas cercanos a Paz sufrieron (realmente) por parte de Bolaño y sus compañeros cuando la etapa real visceralista. Por otra parte, también es cierto que el Bolaño más maduro de la entrevista a Mónica Maristain declara admirar a Monsiváis porque "sigue con las uñas aceradas" (2004: 342) en sus críticas a la sociedad y al mundo literario.
} 
Antes de terminar, cabe destacar una interesante coincidencia que muestra la cercanía entre literatura y vida en la que se movía el autor: justo en 1977, año en el que se sitúan los hechos de la primera parte de Los detectives salvajes, Roberto Bolaño se expresaba de la siguiente forma acerca de la situación de la poesía latinoamericana:

si por panorama general entendemos un movimiento al menos estéticamente al margen del aparato oficial o un subpanorama ética y estéticamente al margen, [...] una interpretación transformadora [...] de una realidad cotidiana sangrienta en donde es imposible verdaderamente crear sin subvertir, en donde es imposible subvertir sin ser apaleado, en donde es imposible ser apaleado sin adoptar [...] posturas de rechazo total a situaciones culturales burguesas [...] el panorama se me presenta como el segundo cartucho de dinamita de la poesía latinoamericana [...] por un lado escriben los jóvenes decentes, los de la cotidianidad de toilette, los caligrafistas, los que buscan un statu de escritor. Por otro están los anarquistas, los poetas narrativos y los nuevos líricos marxistas, los vagabundos, los que viven poesía (Bolaño y Boccanera, 1977: 42-43)9.

Las citadas reflexiones presentan una fuerte afinidad con el panorama que Bolaño satiriza en la novela mencionada, y con ciertas consideraciones de sus personajes. Es evidente el aprecio durante su época de juventud infrarrealista por los que "viven poesía", convicción que hace encarnar, posteriormente, a sus míticos antihéroes realvisceralistas.

Queda claro que siempre, desde sus primeras producciones y a lo largo de toda su trayectoria artística, Bolaño quiso evidenciar no tanto, o no sólo, la importancia de la buena poesía en sentido más general, sino más bien la necesidad de ser poeta en la vida y con la vida para revolucionar así el panorama de las letras: ésta es la única situación que le permite al hombre vivir la existencia plenamente, sin estar encarcelado en ningún esquema social o cultural.

Y queden como confirmación de todo lo que se ha expuesto las palabras de los jóvenes protagonistas mexicanos de Amuleto, cuando expresan su sueño de: "no estar atados a ningún lugar, hacer de nuestras vidas un arte" (Bolaño, 1999: 122).

${ }^{9}$ A este diálogo entre Bolaño y Jorge Alejandro Boccanera alude en la ficción de Los detectives salvajes el personaje de Fabio Ernesto Logiacomo, al igual que Boccanera argentino, ganador del premio Casa de las Américas, con un nombre compuesto y un apellido italiano, quien dice: "lo que querían no era una entrevista sino un conversatorio, un diálogo [...] para publicar en una de las mejores revistas mexicanas. El diálogo iba a ser entre un mexicano [...] un chileno [...] y un argentino, yo [...] Al final el conversatorio sólo lo hicimos entre el chileno y yo" (1998: 150). 


\section{REFERENCIAS}

Roberto, Bolaño. 1976a, "El estridentismo", en Plural 61, pp. 48-50. 1976b. "Tres estridentistas en 1976: Arqueles Vela, Maples Arce, List Arzubide", en Plural 62, pp. 48-60. . 1977. "Déjenlo todo, nuevamente. Primer manifiesto del movimiento infrarrealista”, en Revista INFRA 1, pp. 5-11. (En línea). Disponible en: http://www.infrarrealismo.com. Consulta: 21/09/2009.

. 1979. Muchachos desnudos bajo el arcoiris de fuego. México D.F.: Extemporáneos.

. 1998. Los detectives salvajes. Barcelona: Anagrama.

1999. Amuleto. Barcelona: Anagrama.

2001. Putas asesinas. Barcelona: Anagrama.

2004. Entre paréntesis, Barcelona: Anagrama.

Bolaño, Roberto y Boccanera, Jorge. 1977. "La nueva poesía latinoamericana ¿Crisis o renacimiento?, en Plural 68, pp. 41-49.

Boullosa, Carmen. 2002. "Carmen Boullosa entrevista a Roberto Bolaño", en Manzoni, Celina (ed.). Roberto Bolaño: la escritura como tauromaquia. Buenos Aires: Corregidor, pp. 105-113.

Donoso Pareja, Miguel. 1979. "Once poetas, seis países: ¿Poesía concreta o poesía en proceso?”, prólogo a Roberto Bolaño (antologador). Muchachos desnudos bajo el arcoiris de fuego. México D.F.: Extemporáneos.

Fernández, Teodosio. 1987. La poesía hispanoamericana en el siglo XX. Madrid: Taurus.

Manzoni, Celina (ed.). 2002. Roberto Bolaño: la escritura como tauromaquia. Buenos Aires: Corregidor.

Maples Arce, Manuel. 1991. "Actual 1". En Jorge Schwartz. Las vanguardias latinoamericanas. Textos pragmáticos y críticos. Madrid: Cátedra.

Schwartz, Jorge. 1991. Las vanguardias latinoamericanas. Textos pragmáticos y críticos. Madrid: Cátedra. 